\title{
Calcifications due to Mitral Valve Disease Induced by Rheumatoid Arthritis in K/B.g7 Mice
}

\author{
Elena Konrath, Dr. Bryce Binstadt, M.D., Ph.D \\ University of Minnesota Medical School, Center for Immunology
}

Background and Hypothesis: Rheumatoid arthritis (RA) is a rheumatic autoimmune disease wherein the host generates self-antibodies that target the synovial lining of joints. However, it's been observed that patients with RA also develop Mitral Valve Disease (MVD), although the mechanism is not very well known. In my project, I focused on the physiological changes of the heart during the formation of MVD, specifically focusing on calcifications and whether they form on the inflamed valve. I hypothesized that calcifications are developing during disease progression, resulting in advancement of the inflammation cycle. Experimental Design or Project Methods: A total of thirteen mouse hearts were removed and stored in OTC at -80 C: 5 K/B.g7 (7 months), 5 K/B.g7 (8 weeks) and $3 \mathrm{~B} 6$ (1.5 years). The hearts were sectioned and stained using calcium-staining Alizarin Red S. Additional sections were also stained with Hemotoxylin and Eosin stain.

Results: From my analysis, it was apparent that there were no calcifications on the mitral valves in any of the samples. Pigmentation was apparent, but potential calcium was ruled out when compared to the H\&E stains. There were heavy calcium deposits in the aorta and aortic valve in forty percent of the MVD samples. Additionally, calcium was seen near the apex of the left ventricle in three of the ten MVD samples.

Conclusion and Potential Impact: In conclusion, it doesn't appear that calcification occurs in the mitral valve, neither in the early nor late onset of RA. However, I pursued the calcifications seen in the aorta because of previous research done with calcifications seen in RA mice eating a high-fat diet. Currently, I am investigating further with the entirety of the aorta and staining to see if the calcification is isolated near the valve or if the calcifications spread. I am hoping that my findings will assist in identifying the physiological changes that are seen in rheumatoid arthritis. 